\title{
Trastorno por Déficit de Atención con Hiperactividad: Revisión del tratamiento psicológico
}

\author{
José Antonio López-Villalobos. Complejo Asistencial Universitario de Palencia \\ María Victoria López-Sánchez. Universidad de Santiago de Compostela \\ Jesús Andrés-De Llano. Complejo Asistencial Universitario de Palencia
}

\author{
(i) $0000-0003-1533-628 X$ \\ (iD) $0000-0002-2532-7507$ \\ (iD) $0000-0001-9989-4996$
}

Recepción: 03.11.2018| Aceptado: 11.02.2019

Correspondencia a través de ORCID: José Antonio López Villalobos

Citar: Lopez-Villalobos, JA., Lopez-Sanchez, MV. y Andres-De Llano, J. (2019). Trastorno por Déficit de Atención con Hiperactividad: revisión del tratamiento psicológico. ReiDoCrea, 8, 95-105.

Resumen: El tratamiento del trastorno por déficit de atención con hiperactividad (TDAH) no presenta en el momento actual una solución óptima a medio / largo plazo y se precisa seguir investigando sobre las diferentes opciones terapéuticas en el contexto familiar, social y escolar. El objetivo del estudio es hacer una revisión narrativa actualizada de los tratamientos psicológicos del TDAH en infancia y adolescencia. Método: Se revisan guías de práctica clínica, meta-análisis y revisiones sistemáticas sobre tratamiento de TDAH en los últimos años y en los principales buscadores bibliográficos. Resultados: La terapia de conducta presenta eficacia en el tratamiento de la clínica de TDAH y su comorbilidad, así como la terapia cognitivo conductual integrada en módulos de tratamiento que incluyen terapia de conducta. El neurofeedback y mindfulness se consideran tratamientos prometedores, pero presentan necesidad de más estudios que confirmen su eficacia. El entrenamiento cognitivo se considera un tratamiento con insuficiente evidencia científica. Conclusiones: La terapia conductual y cognitivo conductual son tratamientos eficaces en la mejoría de la clínica de TDAH.

Palabras Clave: Psicoterapia | Psicopatología

\section{Attention Deficit Hyperactivity Disorder: Psychological treatment review}

Abstract: The treatment of attention deficit hyperactivity disorder (ADHD) does not currently offer an optimal medium / long term solution and it is necessary to continue investigating the different therapeutic options in the family, social and school context. The objective of the study is to present an updated narrative review of the psychological treatments of ADHD in childhood and adolescence. Method: We review clinical practice guidelines, meta-analysis and systematic reviews on ADHD treatment in recent years and in the main bibliographic search engines. Results: Behavioral therapy presents evidence of efficacy in the treatment of ADHD and comorbidity as well as cognitive behavioral therapy integrated into treatment modules that include behavioral therapy. Neurofeedback and mindfulness are considered promising treatments, but more studies are necessary to confirm their effectiveness. Cognitive training is considered a treatment with insufficient scientific evidence. Conclusions: Behavioral and Cognitive Behavioral therapy are effective treatments in improving clinical ADHD.

Key words: Psychotherapy | Psychopathology

\section{Introducción}

El objetivo del estudio es revisar la eficacia de los tratamientos psicológicos en niños y adolescentes con trastorno por déficit de atención con hiperactividad (TDAH).

EI TDAH se caracteriza por un patrón persistente de inatención y/o hiperactividad impulsividad que interfiere con el funcionamiento o desarrollo y presenta interferencia en el funcionamiento académico, social o laboral (American Psychiatric Association, 2014). Se considera un trastorno con una demanda clínica creciente, importante afectación en la esfera clínica, social, familiar o académica y una prevalencia a nivel mundial en la infancia y adolescencia de un 5.29\% (Polanczyk, de Lima, Horta, Biederman \& Rohde, 2007). 
En la actualidad el diagnóstico del trastorno se considera básicamente clínico y desde hace varias décadas se ha tratado fundamentalmente con metilfenidato y anfetaminas que con frecuencia producen mejorías en la clínica base del trastorno. Aunque la medicación es eficaz en el tratamiento del TDAH en los ensayos controlados aleatorios a corto / medio plazo (Chan, Fogler \& Hammerness, 2016; Krull, 2017) y está indicada como tratamiento en niños no preescolares y adolescentes, tiene una serie de limitaciones potenciales. Entre estas tenemos la posible respuesta parcial o no respuesta (Faraone, Biederman \&Aleardi, 2006), posibles efectos adversos como disminución del apetito, pérdida de peso, trastornos del sueño, disminución del crecimiento, dolor abdominal, dispepsia, aumento de presión arterial y frecuencia cardíaca, tics, irritabilidad, ansiedad, somnolencia, mareo o dolor de cabeza (Richardson et al., 2015; Storebø et al., 2015; Thapar \& Cooper, 2016), incertidumbre sobre los costos y beneficios a largo plazo (Molina et al., 2009), mala adherencia (Adler \& Nierenberg, 2010) y actitudes negativas relacionadas con la medicación de pacientes, padres o clínicos (Kovshoff et al., 2012). Estas circunstancias y la frecuente recomendación de los tratamientos multimodales (farmacológicos, psicológicos, psicopedagógicos) y multidisciplinares en el tratamiento de TDAH, hacen recomendable una revisión actualizada de los tratamientos psicológicos del trastorno, considerando los nuevos modelos de intervención.

En este contexto nos planteamos como objetivo principal revisar la eficacia de los tratamientos psicológicos no farmacológicos en niños y adolescentes contrastorno por déficit de atención con hiperactividad (TDAH).

\section{Método}

El presente estudio es una revisión narrativa (no sistemática) de la literatura científica disponible en el año 2018 sobre el tratamiento psicológico del TDAH en niños y adolescentes. Para esta revisión se realiza una búsqueda bibliográfica de las principales guías de práctica clínica desarrolladas en los últimos 8 años sobre tratamiento de TDAH en infancia y adolescencia. Además del análisis de estas guías de práctica clínica, se considerarán revisiones sistemáticas y metaanálisis sobre tratamiento psicológico realizados en los últimos cuatro años. El estudio se completará con la consideración de ensayos clínicos e investigaciones cuasi experimentales sobre otras modalidades de intervención psicológica sobre las que aún no existe suficiente contraste científico, pero aportan tendencias que serán objeto de evaluación más consistente en el futuro.

Para la revisión de la literatura sobre tratamientos psicológicos se utilizarán las bases de datos Pubmed/Medline, Psycinfo, Bases del CSIC (sumarios ISOC e IME) y Web of Knowledge. Los criterios de búsqueda bibliográfica en español e inglés son los siguientes términos y sus combinaciones: TDAH, terapia, terapia de conducta, terapia cognitivo conductual, terapia cognitiva, neurofeedback, entrenamiento cognitivo y mindfulness. Se excluirán tratamientos farmacológicos, dietéticos o de ejercicio físico. La búsqueda se limitó a la infancia y adolescencia. La selección final de nuestra revisión incluyó 9 guías de práctica clínica que realizan recomendaciones en función de la calidad metodológica de los estudios incluidos y/o del balance beneficios riesgos mediante sistemas como SIGN o GRADE y 19 revisiones sistemáticas / metaanálisis.

Las 9 guías de práctica clínica revsadas fueron las siguientes: National Institute for Health and Care Excellence (NICE, 2016), Canadian Agency for Drugs and Technologies in Health (CADTH, 2011), American Academy of Pediatrics (AAP) (Wolraich et al., 2011), American Academy of Family Physicians (AAFP) (AAFP, 2016), Guía de práctica clínica sobre intervención terapéutica en TDAH (SNS, 2017), National 
Health and Medical Research Council de Australia (NHMRCA, 2012), ADHD University of Michigan Health System (UMHS, 2013), Danish Health Authority (DHA, 2016) y Canadian ADHD Resource Alliance (CADDRA, 2018).

\section{Resultados}

Estructuraremos este apartado analizando la eficacia de cada una de las principales líneas de tratamiento en el ámbito del TDAH: Terapia de Conducta, Terapia Cognitivo Conductual, Neurofeedback, Entrenamiento Cognitivo y Mindfulness.

\section{Terapia de conducta (TC)}

Utiliza técnicas de refuerzo para conseguir cambios en la conducta y mejorar el control de la actividad motora, impulsividad o la atención. En las familias con hijos que presentan TDAH se suele trabajar aspectos como establecimiento de normas y límites, entrenamiento en hábitos positivos, modificación de conductas disruptivas o entrenamiento en conductas positivas. En la escuela se trabajan dimensiones como realización de las tareas, conductas de organización, habilidades académicas, comportamiento en clase o relación con los compañeros. Se utilizan procedimientos como el refuerzo, coste de respuesta, economías de fichas, tiempo fuera de reforzamiento o sobrecorrección en el contexto del análisis funcional de la conducta problema. Muchos de los tratamientos como el entrenamiento a padres, terapia cognitivo conductual, entrenamiento en habilidades sociales 0 intervenciones psicopedagógicas se basan en los principios de la terapia de conducta.

La mayoría de las guías de práctica clínica recomiendan la TC como primera opción de tratamiento en edad preescolar (AAP, 2011; CADDRA, 2018; NHMRCA, 2012; NICE, 2016; SNS, 2017; UMHS, 2013) y en casos de TDAH escolares sin importante intensidad sintomatológica o importante deterioro funcional (DHA, 2016; NICE, 2016; SNS, 2017). Es habitual que las guías de práctica clínica recomienden la TC en combinación con medicación estimulante en niños no preescolares por el beneficio sobre los síntomas centrales de TDAH (AAP, 2011; DHA, 2016; NHMRCA, 2012; UMHS, 2013). Al respecto, la revisión de Catalá-López (2017) refleja que la combinación de medicación estimulante y TC es más eficaz que la medicación estimulante o no estimulante consideradas de forma aislada y que cualquiera de las intervenciones psicosociales (conductual, neurofeedback, entrenamiento cognitivo).

La Sociedad de Psicología Clínica del Niño y del Adolescente de la División 53 de la American Psychological Association considera la TC como un tratamiento de eficacia probada para el TDAH (nivel 1) en entrenamiento de padres en el manejo de la conducta, comportamiento en el aula, intervenciones conductuales con compañeros o intervenciones combinadas en manejo de conducta (entrenamiento padres, profesores y compañeros) y entrenamiento en organización (Evans, Owens \& Bunford, 2014).

Revisiones sistemáticas y metaanálisis valoran la influencia de la TC en la mejoría de la clínica base de TDAH y en mayor medida en comportamientos asociados (CataláLópez et al., 2017; Chan et al., 2016; Evans et al., 2014; Fabiano, Schatz, Aloe, Chacko \& Chronis-Tuscano, 2015; Hodgson, Hutchinson \& Denson, 2014; Sibley, Kuriyan, Evans, Waxmonsky \& Smith, 2014; Sonuga-Barke et al., 2013; Goode et al., 2018), especialmente en combinación con medicación estimulante, que suele ser la opción más recomendada en el TDAH de edad escolar (Catalá-López et al., 2017, Chan et al., 2016; Krull, 2017). 
En estudiantes preescolares y escolares existe evidencia científica (ensayos clínicos) de eficacia de la TC en el entrenamiento a padres sobre manejo de conducta, en la mejora de los síntomas nucleares del TDAH, problemas de conducta, problemas emocionales y competencia de parental. Varios programas (New Forest Parenting Program, Helping the Noncompliant Child, Positive Parenting Program, Comunication method, Community Parent Education Program o Incredible Years) han demostrado su validez en este margen de edad (CADDRA, 2018; Serrano-Troncoso et al., 2013; SNS, 2017).

En adolescentes una revisión sistemática de Chan et al., (2016) que incluye 2668 participantes observa eficacia de los tratamientos psicosociales (técnicas de entrenamiento conductual, cognitivo-conductual y de entrenamiento en habilidades para padres, profesores y niños) que presentan un tamaño del efecto mediano para los síntomas de TDAH, emocionales o conductuales concurrentes y funcionamiento interpersonal; así como mayor tamaño del efecto para las habilidades académicas y organizacionales.

Finalizamos este apartado sobre la TC valorando su influencia sobre las alteraciones del comportamiento y sobre la disfuncionalidad social o escolar que debe aparecer en la clínica de TDAH para que se considere el trastorno. Se ha comprobado mediante ensayo clínicos que los cumplidores del programa Challenging Horizons Program, vinculado a la TC, mostraron beneficios importantes en la organización, comportamientos disruptivos, desempeño de la tarea y calificaciones, respecto a los controles, con resultados que perduraron 6 meses después del término del tratamiento (Schultz, Evans, Langberg \& Schoemann, 2017). En la misma línea el TC (entrenamiento para padres enfocado en la tarea y un boletín de calificaciones diario) centrado en las tareas escolares presenta beneficios claros para la terminación y exactitud de la tarea de los niños, mientras que la medicación estimulante de acción prolongada dio lugar a efectos limitados y no significativos (Merrill et al., 2017). También se ha demostrado mediante un ensayo clínico que cuando el programa de tratamiento Child Life and Attention Skills involucra conjuntamente a padres, profesores y niños el resultado es mejor que realizado solo con los padres o con el tratamiento habitual. El programa que integra entrenamiento de los padres, consulta del profesor / notas de la escuela-hogar y entrenamiento de habilidades del niño, consiguió mejoras en atención informada por el docente, habilidades de organización, habilidades sociales y funcionamiento global en casos de TDAH inatento (Pfiffner et al., 2014).

En resumen la terapia de conducta ejerce un efecto sobre la clínica de TDAH y en las dimensiones vinculadas al deterioro funcional.

\section{Terapia Cognitivo Conductual (TCC)}

Incluye técnicas como autoinstrucciones, resolución de problemas, inhibición de respuestas o autocontrol y ayuda a desarrollar formas de pensar y comportarse más planificadas y reflexivas, identificando las cogniciones poco adaptativas que son sustituidas por otras más adecuadas (Serrano-Troncoso et al., 2013). La revisión sistemática de Catalá-López et al. (2017), con 48 ensayos sobre intervención psicológica, incluye la TCC en el grupo de terapias de comportamiento valorando su importancia en la mejoría de la clínica base de TDAH y la revisión sistemática de Chan et al. (2016), valora la influencia de tratamientos psicosociales (incluyen TCC) sobre los resultados funcionales, como terminación de la tarea, habilidades organizacionales, síntomas reportados por los padres de TDAH y la psicopatología coexistente. 
En la adolescencia un modelo que incluye la TCC y que integra psicoeducación, habilidades de planificación y organización, entrenamiento en habilidades académicas para reducir la distracción, terapia cognitiva, manejar la tendencia a la dilación, comunicación y manejo emocional, observa disminución del absentismo escolar y mejora en el cumplimiento del horario, disminución de la dosis de medicación estimulante, mejora a nivel conductual notificada por los padres y disminución de la inatención en el aula notificada por los profesores (Antshel \& Olszewski, 2014).

La guía CADDRA (2018), considera la eficacia de la TCC en la clínica de TDAH, aunque valora su mayor eficacia cuando se utiliza conjuntamente con la medicación. El grupo de trabajo para TDAH (SNS, 2017) recomienda como primera opción entre las terapias psicológicas la TC y TCC al considerarlas un referente cuando hablamos de tratamientos empíricamente validados.

Las TCC actuales para el TDAH adulto muestran tamaños de efecto comparables a los tratamientos conductuales para niños con el mismo problema, que se consideran tratamientos bien establecidos (Knouse, Teller \& Brooks, 2017).

\section{Neurofeedback (NF)}

El NF es un tratamiento que proporciona retroalimentación de las señales electroencefalográficas en tiempo real y tiene como objetivo la adquisición de autocontrol sobre determinados patrones de actividad cerebral y la aplicación de estas habilidades en las actividades de la vida diaria.

La División 53 de la American Psychological Association considera el NF como un tratamiento prometedor donde se demuestra que la intervención es mejor que no tratar, pero sin todos los controles metodológicos adecuados (Evans et al., 2014). Revisiones sistemáticas consideran el NF como una intervención prometedora por la mejora en la clínica de TDAH (Cortese et al., 2016; Hodgson et al., 2014; SonugaBarke et al., 2013), aunque su efecto disminuye o desaparece cuando la evaluación de resultados es ciega (Cortese et al., 2016; Sonuga-Barke et al., 2013). Otra revisión sistemática reciente, encuentra ensayos clínicos que en los que el NF disminuye los síntomas de TDAH en la comparación TDAH / controles, aunque los estudios no dejan constancia de seguimiento o generalización (Goode et al., 2018). Estudios de contraste grupo de intervención / placebo y utilización de asignación aleatoria de la intervención no han observado tamaños del efecto significativos en los síntomas de TDAH (CADDRA, 2018).

En síntesis, con la evidencia actual el NF se considera una intervención prometedora, pero no puede recomendarse como intervención basada en evidencia científica para el funcionamiento global y mejora de los síntomas principales del TDAH, hasta que se contraste mediante ensayos clínicos correctamente diseñados (CADDRA, 2018; Catalá-López et al., 2017; Cortese et al., 2016).

\section{Entrenamiento cognitivo (EC) (memoria de trabajo, atención)}

EI EC es habitualmente realizado a través de procedimientos informáticos que gradúan la dificultad de la tarea a lo largo de las sesiones para desafiar al paciente en los límites de su competencia en las áreas cognitivas trabajadas (en el caso de TDAH: memoria de trabajo y atención). La División 53 de la American Psychological Association lo considera como un tratamiento con evidencia no probada (Evans et al., 2014) y existen revisiones sistemáticas que valoran la existencia de un efecto en la clínica total básica de TDAH que disminuye cuando la evaluación es probablemente 
ciega (Cortese et al., 2015; Sonuga-Barke et al., 2013). El entrenamiento es más eficaz cuando se entrenan conjuntamente atención y memoria de trabajo, mejorando la atención y no la hiperactividad; observando que este efecto disminuye cuando la evaluación es probablemente ciega, no siendo eficaz para mejorar el rendimiento académico (Cortese et al., 2015).

Se ha estudiado el entrenamiento en memoria de trabajo mediante un programa de entrenamiento informático (Cogmed Working Memory Training), observándose que mejora la clínica de TDAH en unos estudios pero no en otros, presentando en ambos casos dificultades metodológicas (Chacko et al., 2013). En esta misma línea una revisión de ensayos clínicos y estudios cuasi experimentales observó que el entrenamiento en memoria de trabajo produce discretos efectos de entrenamiento específicos a corto plazo, que no generalizan (Melby-Lervåg \& Hulme, 2013).

La guía clínica Danesa (DHA, 2016) recomienda el entrenamiento cognitivo mediante procedimientos informáticos para mejorar levemente los síntomas de TDAH y considera que se trata de una recomendación débil con nivel de evidencia científica baja.

Una reciente revisión sistemática (Goode et al., 2018) encuentra un ensayo clínico de buena calidad que utilizando el "Cogmed Robo Memo Program" no aprecia diferencias en TDAH entre casos y controles después de 8 meses y otro estudio observacional de baja calidad donde aprecia diferencia a favor del EC.

En resumen, no está clara la influencia del EC en la mejora de la clínica del TDAH y revisiones sistemáticas de ensayos clínicos indican que con la evidencia actual no puede recomendarse como intervención basada en evidencia para el funcionamiento global y mejora de los síntomas principales del TDAH, hasta que se contraste mediante ensayos clínicos correctamente diseñados (Catalá-López et al., 2017; Sibley et al., 2014).

\section{Mindfulness (MF)}

El MF consiste en la práctica formal del aumento de la conciencia, la atención en el momento presente y la observación sin prejuicios, que teóricamente pudiera mejorar la atención y reducir la conducta impulsiva (Kabat-Zinn, 2003). Es una intervención basada en la meditación, que pone el énfasis en la observación y una postura no reactiva hacia los pensamientos, emociones y estados corporales de uno mismo.

Al considerar los diversos tipos de prácticas, dos tipos de meditación son ampliamente reconocidos: atención centrada y receptiva. La meditación concentrada requiere centrarse en un pensamiento específico, como una imagen o una sensación corporal como la respiración. La meditación de vigilancia abierta o receptiva, incluye observar el contenido de la experiencia (por ejemplo, sensaciones, pensamientos y emociones) de un momento a otro sin reaccionar. En la meditación receptiva, la atención se extiende a todo el campo de la conciencia e implica estar alerta a cualquier estímulo que surja en el momento. Al mejorar la atención, el mindfulness puede potencialmente mejorar varios de los principales síntomas del TDAH, como la realización de la tarea, la autorregulación y el control de los impulsos (Modesto-Lowe, Farahmand, Chaplin \& Sarro, 2015).

En una revisión "descriptiva" de estudios cuasi experimentales con adolescentes y adultos con medidas pre - post tratamiento y con serias dificultades metodológicas (algunos estudios sin grupo control, en otros el grupo control es lista de espera, no 
evaluación ciega, no aleatorización, en ocasiones con tratamiento farmacológico, en ocasiones se mezcla la intervención de mindfulness con prácticas de autorregulación... ) se llega a la conclusión de que las intervenciones mediante Mindfulness en casos de TDAH resultan prometedoras pues consiguieron mejorar la sintomatología atencional, hiperactiva e impulsiva (Forcadell, Astals, Treen, Chamorro y Batlle, 2016).

Otra revisión reciente concluye que a pesar de algunas limitaciones metodológicas de los estudios analizados se observa la efectividad del Mindfulness como una alternativa o un complemento al tratamiento del TDAH, mejorando la atención, hiperactividad, impulsividad, regulación emocional e incluso la ansiedad o depresión asociada (Mariño, Cervera y Fernández, 2017). Las limitaciones de los 13 estudios revisados (ausencia de grupo control en 7 estudios, falta de seguimiento, muestras pequeñas y no representativas en 8 estudios, no aleatorización en 7 estudios) y el hecho de que la revisión incluye estudios con niños que tienen un número de casos pequeño (4 estudios con $n<10,2$ con $n<22$ y 1 con $n=120$ ) hace ser cautos a la hora de considerar estas conclusiones como definitivas. Un ensayo clínico cuyo protocolo fue incluido en esta revisión y publicado con posterioridad, presenta una muestra de 100 familias y sus hijos con TDAH (5-7 años), observando reducción significativa de síntomas de hiperactividad, inatención, ansiedad, agresión, problemas internalizantes y externalizantes en los niños y mejoría del estrés parental, así como de su bienestar psicológico (Lo et al., 2017). Este estudio presenta las limitaciones de no controlar el mantenimiento de la mejoría clínica en el tiempo y de no utilizar medidas de resultado ciegas.

La guía CADDRA (2018) refiere que el MF disminuye los síntomas de TDAH e incrementa la autodirección y autorregulación.

En síntesis, la intervención terapéutica resulta prometedora en el ámbito del TDAH, aunque son necesarios ensayos clínicos bien diseñados que permitan recomendar con evidencia científica suficiente el MF como tratamiento consolidado para el TDAH.

\section{Discusión}

Los tratamientos psicológicos ya son considerados relevantes en el ámbito del TDAH. Se consideran la primera opción de tratamiento en edad preescolar (CADDRA, 2018; NICE, 2016) y en la edad escolar para casos con sintomatología leve o escaso deterioro funcional (DHA, 2016; NICE, 2016). Cuando se habla de tratamiento farmacológico para casos con afectación clínica importante, es muy frecuente que la recomendación sea la realización de una intervención combinada con tratamientos psicológicos (Catalá-López et al., 2017; DHA, 2016; Krull, 2017).

A su vez, desde hace tiempo los resultados terapéuticos en TDAH se valoraban en la medida que se reducían los síntomas básicos de inatención, hiperactividad e impulsividad; pero en la actualidad el enfoque clínico y de valoración de una intervención terapéutica se está moviendo hacia la mejora del deterioro funcional y calidad de vida de los casos con TDAH (CADDRA, 2018). En estas dimensiones los tratamientos psicológicos han mostrado mayor eficacia (Catalá-López et al., 2017; Chan et al., 2016).

Nuestra revisión narrativa se ha centrado en tratamientos psicológicos que requieren de un correcto diagnóstico clínico del trastorno y de una adecuada evaluación funcional de las conductas problema que nos permitan plantear con claridad los objetivos de la intervención. Ignorar este apartado impedirá que cualquier intervención 
tenga adecuados resultados, con independencia de su evidencia científica. El uso inadecuado y poco profesional de buenos procedimientos terapéuticos, no garantía buenos resultados.

En este contexto, nuestra revisión ha observado que la terapia de conducta en formato de entrenamiento a padres, profesores o integrada en un grupo más amplio de paquetes terapéuticos, es eficaz en la mejora de la clínica básica de TDAH y en mayor medida en el deterioro funcional asociado y en la comorbilidad (Catalá-López et al., 2017; Evans et al., 2014; Fabiano et al., 2015). La terapia de conducta es generalmente recomendada por las guías clínicas en edad preescolar como tratamiento único y en edades posteriores como tratamiento conjunto con la medicación cuando la clínica y deterioro funcional es relevante o de forma aislada cuando ambas dimensiones presentan menor intensidad (CADDRA, 2018; NICE, 2016). A su vez, la terapia cognitivo conductual se ha mostrado útil cuando es integrada en un paquete de intervenciones más amplio, que suele incluir la terapia de conducta (CADDRA, 2018; Catalá-López et al., 2017; SNS, 2017).

El entrenamiento cognitivo no puede recomendarse como intervención basada en evidencia para el funcionamiento global y mejora de los síntomas principales del TDAH (Catalá-López et al., 2017; Cortese et al., 2015; Goode et al., 2018), aunque existen unos pocos estudios con baja calidad metodológica que muestran relativa mejoría clínica (Chacko et al., 2013).

El neurofeedback puede considerarse como una intervención prometedora en el ámbito del TDAH por su eficacia en la mejora de sus síntomas básicos del TDAH (Cortese et al., 2016; Evans et al., 2014), pero las limitaciones metodológicas de los estudios y resultados contradictorios nos hacen indicar que aún precisamos de ensayos clínicos bien diseñados que confirmen su promesa de utilidad (Catalá-López et al., 2017).

El mindfulness también puede considerarse una intervención prometedora en el ámbito del TDAH por su eficacia en la mejora de los síntomas básicos del TDAH (Lo et al., 2017), pero por las importantes limitaciones metodológicas de muchos estudios y resultados contradictorios, aún precisamos de ensayos clínicos bien diseñados que confirmen su promesa de utilidad (Mariño et al., 2017).

El tratamiento del TDAH suele incluir diversidad de técnicas (psicológicas, farmacológicas, pedagógicas), es multidimensional (familiar, escolar, social) y frecuentemente multiprofesional (médicos, psicólogos, profesores, pedagogos...). La combinación coordinada de estas medidas es la que probablemente garantiza la mejoría sintomatológica y funcional de los casos de TDAH. Nuestra revisión ha valorado algunas de estas dimensiones pero indudablemente está lejos de considerarlas en su totalidad y/o en combinación, como no lo hace ninguna de las revisiones sistemáticas. La reflexión narrativa sobre estas dimensiones sigue siendo necesaria.

Los resultados de nuestra revisión pueden hacer pensar que los procedimientos que no tienen suficiente evidencia científica no funcionan. La realidad no es esa, solo quiere decir que no existe suficiente evidencia científica mediante ensayos clínicos bien diseñados con una muestra representativa y con el tamaño suficiente para contrastar la hipótesis de eficacia.

Nuestros resultados tampoco hacen mención a otros modelos terapéuticos que están siendo utilizados en el ámbito de TDAH. Si nuestra revisión no incluye otros modelos 
es porque no existe suficiente evidencia científica de resultados (ni a favor, ni en contra).

En resumen, existen modelos de intervención con evidencia de eficacia en la clínica de TDAH como la terapia de conducta apoyada ocasionalmente con intervenciones cognitivas y otros que no tienen esa evidencia, pero que pueden tener utilidad. El desarrollo de nuevas investigaciones quizás nos permita ser más precisos en cuanto a nuestra valoración de las prometedoras intervenciones basadas en neurofeedback o mindfulness $y / o$ de otras intervenciones que pudieran surgir.

En todo caso, considerar los resultados de nuestra revisión y tener la mente abierta a otras opciones terapéuticas es una manera de evolucionar en el conocimiento científico. El tratamiento eficaz del TDAH, con estudios que realicen un seguimiento prolongado de resultados y se enfoquen en la mejora del deterioro funcional y calidad de vida de los afectados es un tema en el que queda camino por andar.

\section{Referencias}

Adler, LD., \& Nierenberg, AA. (2010). Review of medication adherence in children and adults with ADHD. Postgraduate medicine, 122(1), 184-191.

American Academy of Family Physicians (AAFP) (2016). ADHD in Children and Adolescents. Clinical Practice Guideline. Disponible en https://www.aafp.org/patient-care/clinicalrecommendations/all/ADHD.html.

American Psychiatric Association (2014). Manual diagnóstico y estadístico de los trastornos mentales DSM -5. Madrid: Editorial Médica Panamericana.

Antshel, KM., \& Olszewski, AK. (2014). Cognitive behavioral therapy for adolescents with ADHD. Child and adolescent psychiatric clinics of North America, 23(4), 825-842.

Canadian ADHD Resource Alliance (2018). Canadian ADHD Practice Guidelines. Toronto: CADDRA.

Canadian Agency for Drugs and Technologies in Health (2011). Guidelines and Recommendations for ADHD in Children and Adolescents. Disponible https://www.ncbi.nlm.nih.gov/books/NBK174625/

Catalá-López, F., Hutton, B., Núñez-Beltrán, A., Mayhew, AD., Page, MJ., Ridao, M., et al. (2017). The pharmacological and non-pharmacological treatment of attention deficit hyperactivity disorder in children and adolescents: protocol for a systematic review and network meta-analysis of randomized controlled trials. PLOS ONE, 12(7), 1-31.

Chacko, A., Feirsen, N., Bedard, AC., Marks, D., Uderman, JZ., \& Chimiklis, A. (2013). Cogmed working memory training for youth with ADHD: a closer examination of efficacy utilizing evidence-based criteria. Journal of Clinical Child \& Adolescent Psychology, 42(6), 769-783.

Chan, E., Fogler, JM., \& Hammerness, PG. (2016). Treatment of attention deficit hyperactivity disorder in adolescents: a systematic review. Jama, 315(18), 1997-2008.
Cortese, S., Ferrin, M., Brandeis, D., Buitelaar, J., Daley, D., Dittmann, RW. et al. (2015). Cognitive training for attentiondeficit/hyperactivity disorder: meta-analysis of clinical and neuropsychological outcomes from randomized controlled trials. Journal of the American Academy of Child and Adolescent Psychiatry, 54(3), 164-174

Cortese, S., Ferrin, M., Brandeis, D., Holtmann, M., Aggensteiner, P., Daley, D. et al. (2016). Neurofeedback for attentiondeficit/hyperactivity disorder: meta-analysis of clinical and neuropsychological outcomes from randomized controlled trials. Journal of the American Academy of Child \& Adolescent Psychiatry, 55(6), 444-455.

Danish Health Authority (2016). National clinical guideline for the assessment and treatment of $A D H D$ in children and adolescents. Disponible en https://www.sst.dk/en/publications/2014/ /media/8EC150A 85B884908972677B4A4111EA9.ashx.

Evans, SW., Owens, JS., \& Bunford, N. (2014). Evidence-based psychosocial treatments for children and adolescents with attention-deficit/hyperactivity disorder. Journal of Clinical Child \& Adolescent Psychology, 43(4), 527-551.

Fabiano, GA., Schatz, NK., Aloe, AM., Chacko, A., \& ChronisTuscano, A. (2015). A systematic review of meta-analyses of psychosocial treatment for attention-deficit/hyperactivity disorder. Clinical child and family psychology review, 18(1), 77-97.

Faraone, SV., Biederman, J., Spencer, TJ., \& Aleardi, M. (2006). Comparing the efficacy of medications for ADHD using metaanalysis. Medscape General Medicine, 8(4), 4.

Forcadell, E., Astals, M., Treen, D., Chamorro, J., \& Batlle, S. (2016). Entrenamiento en Mindfulness para Pacientes con Trastorno por Déficit de Atención con Hiperactividad (TDAH): una Revisión Descriptiva. Revista de Psicoterapia, 27(103), 203-213. 
Goode, AP., Coeytaux, RR., Maslow, GR., Davis, N., Hill, S., Namdari, B., et al. (2018). Nonpharmacologic Treatments for Attention-Deficit/Hyperactivity Disorder: A Systematic Review. Pediatrics, e20180094.

Grupo de trabajo de la Guía de Práctica Clínica sobre las Intervenciones Terapéuticas en el Trastorno por Déficit de Atención con Hiperactividad (2017). Guía de Práctica Clínica sobre las Intervenciones Terapéuticas en el Trastorno por Déficit de Atención con Hiperactividad (TDAH). Ministerio de Sanidad, Servicios Sociales e Igualdad. Instituto Aragonés de Ciencias de la Salud (IACS). Guías de Práctica Clínica en el SNS

Hodgson, K., Hutchinson, AD., \& Denson, L. (2014). Nonpharmacological treatments for ADHD: a meta-analytic review. Journal of attention disorders, 18(4), 275-282.

Kabat-Zinn, J. (2003). Mindfulness-based interventions in context: past, present, and future. Clinical psychology: Science and practice, 10(2), 144-156.

Knouse, LE., Teller, J., \& Brooks, MA. (2017). Meta-analysis of cognitive-behavioral treatments for adult ADHD. Journal of Consulting and Clinical Psychology, 85(7), 737-750.

Kovshoff, H., Williams, S., Vrijens, M., Danckaerts, M., Thompson, M., Yardley, L., et al. (2012). The decisions regarding ADHD management (DRAMa) study: uncertainties and complexities in assessment, diagnosis and treatment, from the clinician's point of view. European child \& adolescent psychiatry, 21(2), 87-99.

Krull, KR. (2017). Attention deficit hyperactivity disorder in children and adolescents: Overview of treatment and prognosis. UpToDate. Waltham, MA: UpToDate.

Lo, HH., Wong, SW., Wong, JY., Yeung, JW., Snel, E., \& Wong, SY. (2017). The Effects of Family-Based Mindfulness Intervention on ADHD Symptomology in Young Children and Their Parents: A Randomized Control Trial. Journal of attention disorders, DOI: 10.1177/10870547177433.

Mariño, V. Sanz, P. y Fernández, MI. (2017). Efectividad del minfulness en personas con TDAH: estudio de revisión. ReiDoCrea, 6, 260-273.

Melby-Lervåg, M., \& Hulme, C. (2013). Is working memory training effective? A meta-analytic review. Developmental psychology, 49(2), 270.

Merrill, BM., Morrow, AS., Altszuler, AR., Macphee, FL., Gnagy, EM., Greiner, AR., et al. (2017). Improving homework performance among children with ADHD: A randomized clinical trial. Journal of consulting and clinical psychology, 85(2), 111

Modesto-Lowe, V., Farahmand, P., Chaplin, M., \& Sarro, L. (2015). Does mindfulness meditation improve attention in attention deficit hyperactivity disorder? World journal of psychiatry, $5(4), 397$

Molina, BS., Hinshaw, SP., Swanson, JM., Arnold, LE., Vitiello, B., Jensen, PS., et al (2009). The MTA at 8 years: prospective follow-up of children treated for combined-type ADHD in a multisite study. Journal of the American Academy of Child \& Adolescent Psychiatry, 48(5), 484-500.
National Health and Medical Research Council (2012).Clinical Practice Points on the diagnosis, assessment and management of Attention Deficit Hyperactivity Disorder in children and adolescents. Common wealth of Australia. Disponible en https://www.nhmrc.gov.au/_files_nhmrc/publications/attachme nts/mh26_adhd_cpp_2012_120903.pdf

National Institute for Health and Care Excellence (2016). Attention Deficit Hyperactivity Disorder: Diagnosis and Management of $A D H D$ in Children, Young People and Adults. Disponible en https://www.nice.org.uk/ guidance/cg72.

Pfiffner, LJ., Hinshaw, SP., Owens, E., Zalecki, C., Kaiser, NM., Villodas, M., et al. (2014). A two-site randomized clinical trial of integrated psychosocial treatment for ADHD-inattentive type. Journal of consulting and clinical psychology, 82(6), 1115.

Polanczyk, G., de Lima, MS., Horta, BL., Biederman, J., \& Rohde, LA. (2007). The worldwide prevalence of ADHD: a systematic review and metaregression analysis. American Journal of Psychiatry, 164(6), 942-948.

Richardson, M., Moore, DA., Gwernan-Jones, R., ThompsonCoon, J., Ukoumunne, O., Rogers, M. et al. (2015). Nonpharmacological interventions for attentiondeficithyperactivity disorder (ADHD) delivered in school settings: systematic reviews of quantitative and qualitative research. Health technology assessment, 19(45), 1- 470.

Schultz, BK., Evans, SW., Langberg, JM., \& Schoemann, AM. (2017). Outcomes for adolescents who comply with long-term psychosocial treatment for ADHD. Journal of consulting and clinical psychology, 85(3), 250.

Serrano-Troncoso, E., Guidi, M. y Alda-Díez, JA. (2013). ¿Es el tratamiento psicológico eficaz para el trastorno por déficit de atención con hiperactividad (TDAH)? Revisión sobre los tratamientos no farmacológicos en niños y adolescentes con TDAH. Actas Españolas de Psiquiatría, 41(1), 44-51.

Sibley, MH., Kuriyan, AB., Evans, SW., Waxmonsky, JG., \& Smith, BH. (2014). Pharmacological and psychosocial treatments for adolescents with ADHD: An updated systematic review of the literature. Clinical Psychology Review, 34(3), 218-232.

Sonuga-Barke, EJ., Brandeis, D., Cortese, S., Daley, D., Ferrin, M., Holtmann, M., et al. (2013). Nonpharmacological interventions for ADHD: systematic review and meta-analyses of randomized controlled trials of dietary and psychological treatments. American Journal of Psychiatry, 170(3), 275-289.

Storebø, OJ., Krogh, HB., Ramstad, E., Moreira-Maia, CR., Holmskov, M., Skoog, M., et al. (2015). Methylphenidate for attention-deficit/hyperactivity disorder in children and adolescents: Cochrane systematic review with meta-analyses and trial sequential analyses of randomised clinical trials. BMJ, 351, h5203, 1-14.

Thapar, A., \& Cooper, M. (2016). Attention deficit hyperactivity disorder. Lancet, 387 (10024), 1240-50.

University of Michigan Health System (2013). Attention-deficit hyperactivity disorder. Ann Arbor (MI): University of Michigan Health System. Disponible en http://www.med.umich.edu/1info/FHP/practiceguides ladhd/adhd.pdf. 
Wolraich, M., Brown, L., Brown, RT., DuPaul, G., Earls, M., \& Feldman, HM. (2011). Subcommittee on AttentionDeficit/Hyperactivity Disorder; Steering Committee on Quality Improvement and Management. ADHD: clinical practice guideline for the diagnosis, evaluation, and treatment of attention-deficit/hyperactivity disorder in children and adolescents. Pediatrics, 128(5), 1007-1022. 